\title{
ACCURACY ESTIMATION OF THE APPROXIMATED ATANGANA-BALEANU OPERATOR
}

\author{
Krzysztof Oprzedkiewicz, Wojciech Mitkowski \\ Department of Automatics and Robotics, AGH University \\ Krakow, Poland \\ kop@agh.edu.pl,wojciech.mitkowski@agh.edu.pl
}

Received: 12 October 2019; Accepted: 21 January 2020

\begin{abstract}
In the paper, the accuracy analysis of the approximation of the Atangana-Baleanu (AB) operator is presented. The $\mathrm{AB}$ operator is the nonsingular kernel operator proposed by Atangana and Baleanu. It is obtained by replacing the exponential function in the Caputo-Fabrizio operator by the Mittag-Leffler function. The Laplace transform of the AB operator requires approximating the factor $s^{\alpha}$. This is done using the well-known Oustaloup Recursive Approximaion (ORA) approximation. The step and frequency responses of the approximation are compared to the analytical responses. As the cost function, the FIT function available in MATLAB was applied. Results of simulations show that the use of ORA allows us to obtain the accurate approximant of the $A B$ operator.
\end{abstract}

MSC 2010: 45P05, 47B34, $11 \mathrm{~J} 91$

Keywords: Atangana-Baleanu operator, Caputo operator, ORA approximation, nonsingular kernel operator, Mittag-Leffler function

\section{Introduction}

The fractional order (FO) operator using the Atangana-Baleanu (AB) derivative has been proposed in the paper [1]. Its properties are analyzed in [2]. This operator is employed to model of some physical phenomena, mainly diffusion and heat transfer. Examples of its application cover, for example, the analytical solution of the Hristov diffusion equation [3], a number of interesting recent results is collected in the book [4].

Calculations executed with the use of $\mathrm{AB}$ operator require to employing an approximation of $s^{\alpha}$ element. This can be done using different methods, in the time-continuous case, the known Oustaloup approximation (ORA) can be used.

This paper deals with the accuracy analysis while using ORA approximation to express the $\mathrm{AB}$ operator. As a reference, the analytical formula of the step response is used, analogically, as it was done in [5,6].

The paper is organized as follows: preliminaries describe the Atangana-Baleanu operator and its Laplace transform. Next, the analytical formula of the step response 
and the ORA based approximation are proposed. Frequency responses are discussed too. Finally, the simulations illustrating the dependence between accuracy of the approximation and ORA parameters are given.

\section{Preliminaries}

A presentation of elementary ideas is started with a definition of a fractional order, and integro-differential operator given for example by [7]:

Definition 1 (The elementary fractional order operator) The fractional order integrodifferential operator is defined as follows:

$$
{ }_{a} D_{t}^{\alpha} f(t)=\left\{\begin{array}{l}
\frac{d^{\alpha} f(t)}{d t^{\alpha}} \quad \alpha>0 \\
1 \quad \alpha=0 \\
\int_{a}^{t} f(\tau)(d \tau)^{-\alpha} \quad \alpha<0
\end{array} .\right.
$$

where $a$ and $t$ denote time limits of operator calculation, $\alpha \in \mathbb{R}$ denotes the fractional order of the operation.

Next, an idea of the Gamma Euler function is recalled (see for example [8]):

Definition 2 The Gamma function

$$
\Gamma(x)=\int_{0}^{\infty} t^{x-1} e^{-t} d t .
$$

An idea of Mittag-Leffler function needs to be given next. It is a non-integer order generalization of exponential function $e^{\lambda t}$ and it plays crucial role in solution of fractional order state equation. The one parameter Mittag-Leffler function is defined as follows (see for example [8]):

Definition 3 The one parameter Mittag-Leffler function

$$
E_{\alpha}(x)=\sum_{k=0}^{\infty} \frac{x^{k}}{\Gamma(k \alpha+1)} .
$$

The fractional order derivative Atangana-Baleanu operator is obtained via replacing the exponential kernel in the Caputo-Fabrizio (CF) operator by the Mittag-Leffler kernel. It is defined using the Caputo (C) or Riemann-Liouville (RL) definition of the fractional order derivative. Using these definitions, we obtain the Atangana-Baleanu-Caputo (ABC) or Atangana-Baleanu-Riemann (ABR) operator respectively [1]: 
Definition 4 (The Atangana-Baleanu-Caputo (ABC) operator)

$$
{ }_{A B C}{ }_{a} D_{t}^{\alpha}(f(t))=M_{\alpha} \int_{a}^{t} f^{\prime}(x) E_{\alpha}\left(-\alpha \frac{(t-x)^{\alpha}}{1-\alpha}\right) d x .
$$

where $E_{\alpha}(.$.$) is the one parameter Mittag-Leffler function, M_{\alpha}$ is the normalization function equal:

$$
M_{\alpha}=1-\alpha+\frac{\alpha}{\Gamma(\alpha)} .
$$

In (5) $\Gamma(.$.$) is the Gamma function.$

Definition 5 (The Atangana-Baleanu-Riemann (ABR) operator)

$$
{ }_{a}^{A B R} D_{t}^{\alpha}(f(t))=M_{\alpha} \frac{d}{d t} \int_{a}^{t} f(x) E_{\alpha}\left(-\alpha \frac{(t-x)^{\alpha}}{1-\alpha}\right) d x .
$$

where $E_{\alpha}(.$.$) is the one parameter Mittag-Leffler function, M_{\alpha}$ is the normalization function expressed by (5), $\Gamma(.$.$) is the Gamma function.$

The Laplace transforms for the $\mathrm{ABC}$ and $\mathrm{ABR}$ derivatives are as follows:

Definition 6 (The Laplace transform of the $\mathrm{ABC}$ operator)

$$
{ }^{A B C} G(s)=\mathscr{L}\left\{{ }^{A B C}{ }_{a} D_{t}^{\alpha}(f(t))\right\}(s)=\frac{M_{\alpha}}{1-\alpha} \frac{s^{\alpha}\{f(t)\}(s)-s^{\alpha-1} f(0)}{s^{\alpha}+\frac{\alpha}{1-\alpha}} .
$$

Definition 7 (The Laplace transform of the ABR operator)

$$
{ }^{A B R} G(s)=\mathscr{L}\left\{{ }^{A B R}{ }_{a} D_{t}^{\alpha}(f(t))\right\}(s)=\frac{M_{\alpha}}{1-\alpha} \frac{s^{\alpha}\{f(t)\}(s)}{s^{\alpha}+\frac{\alpha}{1-\alpha}} .
$$

For the homogeneous initial condition: $f(0)=0$ both of the Laplace transforms are equal:

$$
\mathscr{L}\left\{{ }^{A B R}{ }_{a} D_{t}^{\alpha}(f(t))\right\}(s)=\mathscr{L}\left\{{ }^{A B C}{ }_{a} D_{t}^{\alpha}(f(t))\right\}(s)=G(s) .
$$

Consequently, the transfer function of the $\mathrm{AB}$ operator takes the following form:

$$
G(s)=\frac{M_{\alpha}}{1-\alpha} \frac{s^{\alpha}}{s^{\alpha}+\frac{\alpha}{1-\alpha}} .
$$

The method proposed by Oustaloup (see [9]) allows us to approximate an elementary FO transfer function $s^{\alpha}$ by the finite, integer-order transfer function, close to Pade approximation:

$$
s^{\alpha} \approx k_{f} \prod_{n=1}^{N} \frac{1+\frac{s}{\mu_{n}}}{1+\frac{s}{v_{n}}}=\frac{L_{O R A}(s)}{D_{O R A}(s)} .
$$


In (11) $N$ is the order of the approximation, $k_{f}$ is the steady state gain. It allows to fit the step response of the approximation to step response of the approximated element $s^{\alpha} . \mu_{n}$ and $v_{n}$ are calculated as follows:

$$
\begin{array}{r}
\mu_{1}=\omega_{l} \sqrt{\eta} \\
v_{n}=\mu_{n} \gamma, n=1, . ., N \\
\mu_{n+1}=v_{n} \eta, n=1, . ., N-1
\end{array}
$$

where:

$$
\begin{gathered}
\gamma=\left(\frac{\omega_{h}}{\omega_{l}}\right)^{\frac{\alpha}{N}} \\
\eta=\left(\frac{\omega_{h}}{\omega_{l}}\right)^{\frac{1-\alpha}{N}}
\end{gathered}
$$

In (13), $\omega_{l}$ and $\omega_{h}$ describe the bandwidth, for which parameters are calculated. During further considerations, it will be expressed in the logarithmic scale:

$$
\begin{gathered}
\omega_{l}=10^{-W}, \\
\omega_{h}=10^{W}
\end{gathered}
$$

where $W \in \mathbb{Z}$ describes the bandwidth.

The use of ORA approximation (11) to (7) or (8) allows us obtain the approximation of the operator. It is as follows:

$$
\mathscr{L}\left\{{ }_{a}^{A B R} D_{t}^{\alpha}(f(t))\right\}(s)=\mathscr{L}\left\{{ }^{A B C}{ }_{a} D_{t}^{\alpha}(f(t))\right\}(s) \approx \frac{L_{O R A}(s)}{\alpha_{1} L_{O R A}(s)+\alpha D_{O R A}(s)} F(s) .
$$

Consequently, the transfer function of the approximation takes the following form:

$$
G_{O R A}(s)=\frac{L_{O R A}(s)}{\alpha_{1} L_{O R A}(s)+\alpha D_{O R A}(s)} .
$$

In (15), (16): $\alpha_{1}=1-\alpha$.

\section{The step responses of the $A B$ operator}

The step response of the Laplace transform (7) or (8) is described by the following remark:

Remark 1 (The step response of the AB operator)

Assume that the Laplace transform of the $\mathrm{ABC} / \mathrm{R}$ operator with an initial condition equal to zero is given by (8). Its step response is equal: 


$$
y(t)=\frac{M_{\alpha}}{\alpha_{1}} E_{\alpha}\left(-\frac{\alpha}{\alpha_{1}} t^{\alpha}\right)
$$

where $E_{\alpha}(.$.$) is the Mittag-Leffler function (3), M_{\alpha}$ is expressed by (5), $\alpha_{1}=1-\alpha \cdot \square$ PROOF The step response is expressed as the following inverse Laplace transform:

$$
\begin{array}{r}
y(t)=\mathscr{L}^{-1}\left\{M_{\alpha} \frac{1}{s} \frac{s^{\alpha}}{\alpha_{1} s^{\alpha}+\alpha}\right\}= \\
\frac{M_{\alpha}}{\alpha_{1}} \mathscr{L}^{-1}\left\{\frac{1}{s} \frac{s^{\alpha}}{s^{\alpha}+\frac{\alpha}{\alpha_{1}}}\right\}
\end{array}
$$

The inverse Laplace transform from (18) is given in the book [10], page 11, Eq (1.35). The use of this equation gives the step response (17) directly, and the proof is finished.

Using (15) or (16), the approximated step response is as follows:

$$
y_{a}(t)=\mathscr{L}^{-1}\left\{\frac{1}{s} \frac{L_{O R A}(s)}{\alpha_{1} L_{O R A}(s)+\alpha D_{O R A}(s)}\right\} .
$$

\section{The frequency responses of the $A B$ operator}

The exact spectral transfer function of the operator is obtained via replacing $s=j \omega$ in (10) and recalling that:

$$
(j \omega)^{\alpha}=\omega^{\alpha}\left(\cos \left(\frac{\alpha \pi}{2}\right)+j \sin \left(\frac{\alpha \pi}{2}\right)\right) .
$$

It is as follows:

$$
G(j \omega)=\frac{M_{\alpha}}{\alpha_{1}}(P(\omega)+j Q(\omega)) .
$$

where $P(\omega)$ and $Q(\omega)$ denote real and imaginary parts of the (21):

$$
\begin{aligned}
& P(\omega)=\frac{1+\frac{\hat{\alpha}}{\omega^{\alpha}} \cos \left(\frac{\alpha \pi}{2}\right)}{1+2 \frac{\hat{\alpha}}{\omega^{\alpha}} \cos \left(\frac{\alpha \pi}{2}\right)+\left(\frac{\hat{\alpha}}{\omega^{\alpha}}\right)^{2}} \\
& Q(\omega)=\frac{\frac{\hat{\alpha}}{\omega^{\alpha}} \sin \left(\frac{\alpha \pi}{2}\right)}{1+2 \frac{\hat{\alpha}}{\omega^{\alpha}} \cos \left(\frac{\alpha \pi}{2}\right)+\left(\frac{\hat{\alpha}}{\omega^{\alpha}}\right)^{2}}
\end{aligned}
$$

The magnitude $M(\omega)$ and phase $\phi(\omega)$ of spectral transfer function (21) are as follows:

$$
M(\omega)=\frac{M_{\alpha}}{\alpha_{1}} \frac{1}{\sqrt{1+2 \frac{\hat{\alpha}}{\omega^{\alpha}} \cos \left(\frac{\alpha \pi}{2}\right)+\left(\frac{\hat{\alpha}}{\omega^{\alpha}}\right)^{2}}} .
$$




$$
\begin{gathered}
M_{d B}(\omega)=20 \log \left(\frac{M_{\alpha}}{\alpha_{1}}\right)-10 \log \left(1+2 \frac{\hat{\alpha}}{\omega^{\alpha}} \cos \left(\frac{\alpha \pi}{2}\right)+\left(\frac{\hat{\alpha}}{\omega^{\alpha}}\right)^{2}\right)[d B] . \\
\phi(\omega)=\arctan \left(\frac{\sin \left(\frac{\alpha \pi}{2}\right)}{\frac{\omega^{\alpha}}{\hat{\alpha}}+\cos \left(\frac{\alpha \pi}{2}\right)}\right) .
\end{gathered}
$$

In (22)-(25) $\hat{\alpha}=\frac{\alpha}{1-\alpha}$. The approximated frequency response is obtained using spectral transfer function $G_{O R A}(j \omega)$ :

$$
G_{O R A}(j \omega)=\frac{L_{O R A}(j \omega)}{\alpha_{1} L_{O R A}(j \omega)+\alpha D_{O R A}(j \omega)} .
$$

\section{Simulations}

The performance of the approximation is estimated using the following cost function, describing the fitting of the approximated result $y_{a}$ to the analytical one $y$. The results can be time or frequency functions.

$$
F I T=\frac{\left\|y-y_{a}\right\|}{\left\|y-\overline{y_{a}}\right\|} .
$$

In (27) $\overline{y_{a}}$ is the average value from approximated result $y_{a}$. The function (27) can be also expressed in \%, as it is done in the MATLAB functions compare and tfest:

$$
F I T_{100}=(1-F I T) \cdot 100 \% .
$$

Firstly, the step responses were analyzed. The analytical step response $y$ was calculated using (17), the approximation $y_{a}$ was calculated using (19) and MATLAB function step. Comparison of the analytical vs approximated step response is shown in Figure 1. The cost function (28) as a function of ORA approximation order $N$ for different non integer orders $\alpha$ is shown in Figure 2, the cost function (28) as a function of non integer order $\alpha$ for different bandwidth $W$ is illustrated by Figure 3 .

Fitting the module of approximation to the exact one expressed by (24) as a function of non integer order $\alpha$ for different bandwidth $W$ is illustrated by Figure 5 .

It can be noted that in the time domain, the accuracy of the approximation depends on the non integer order $\alpha$. For $0<\alpha<0.5$ the approximation is significantly worse than for $0.5<\alpha<1.0$. Next, the good accuracy is achieved for relatively low order $N$ of ORA approximation: the fitting over $99.5 \%$ is assured by $N=6$ and $\alpha>0.5$. Furthermore, the good accuracy requires applying the higher bandwidth, $W=3,4$, but the narrow bandwidth $W=1$ assures the fitting of over $90 \%$ too.

Next, the frequency responses were examined. The exact frequency response was calculated using analytical formulae (21)-(25). The frequency response of approximation was obtained using Nyquist and Bode functions available in MATLAB. 


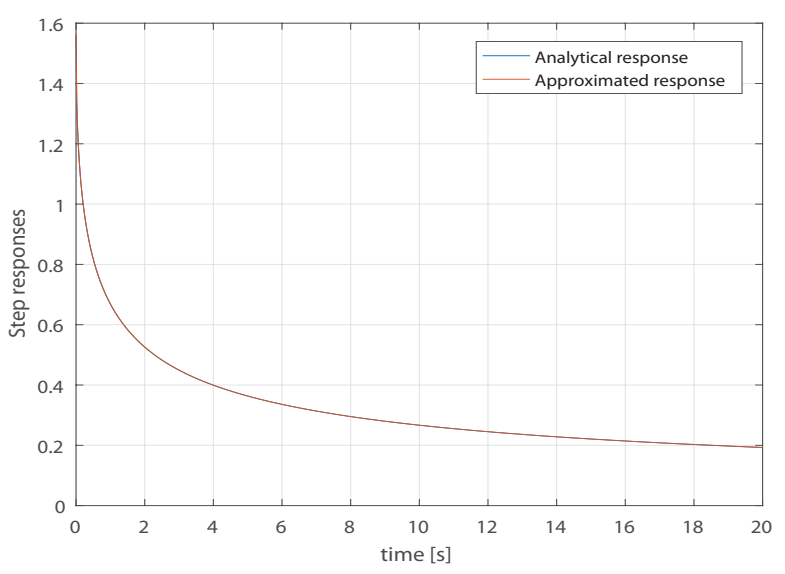

Fig. 1. The comparison of approximated (19) to analytical step response (17) for $\alpha=0.5, N=8, W=4$

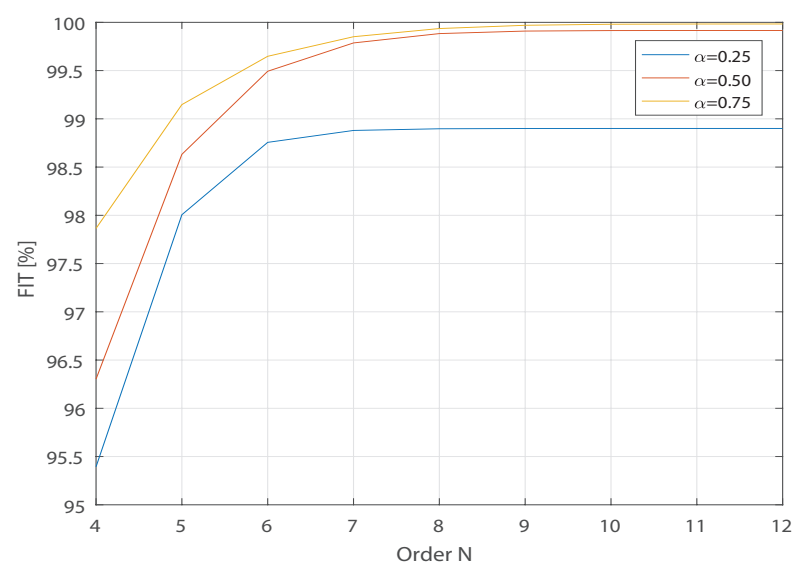

Fig. 2. The fitting of approximation (19) to analytical solution (17) as a function of ORA approximation order $N$ for different orders $\alpha$, the bandwidth $W=4$

The comparison exact vs approximated Nyquist plot is shown in Figure 4, the cost function (28) as a function of $\alpha$ and $N$ is illustrated by Figures 5 and 6 . 


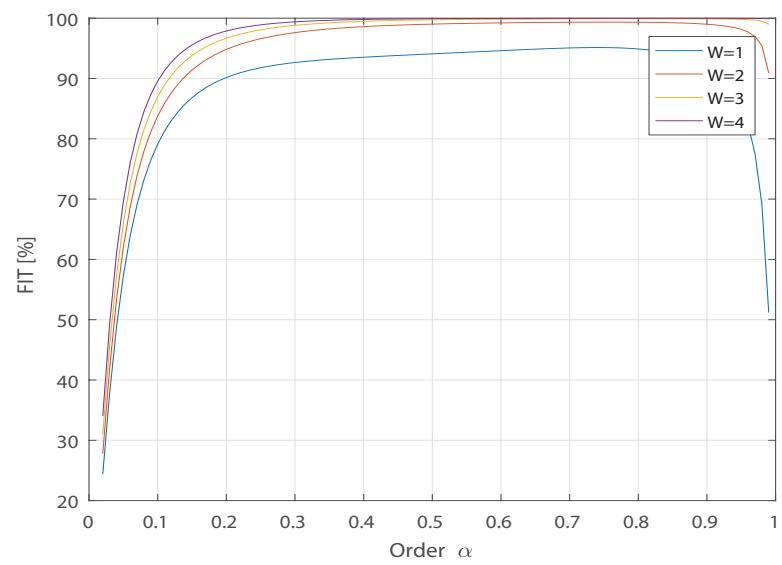

Fig. 3. The fitting of approximation (19) to analytical solution (17) as a function of non integer order $\alpha$ for different bandwidth $W$, the ORA approximation order $N=8$

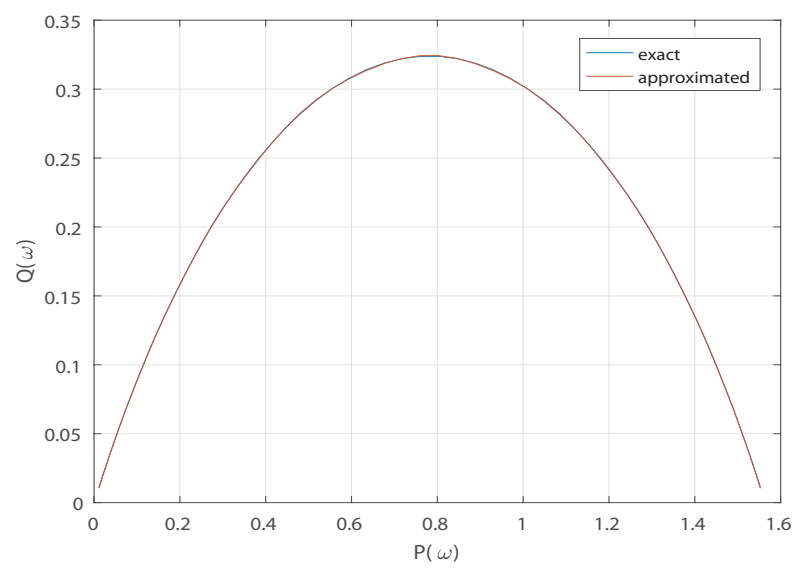

Fig. 4. The comparing of Nyquist diagram of approximation to analytical one (22) for $\alpha=0.5, N=8, W=4$

In the frequency domain, the dependency accuracy on order $N$ (see diagram 6) is analogical as for the time domain. However, the narrow bandwidth $W$ does not cause significant decreasing of the quality of approximation, because it is over $98 \%$ for each $\alpha$. 


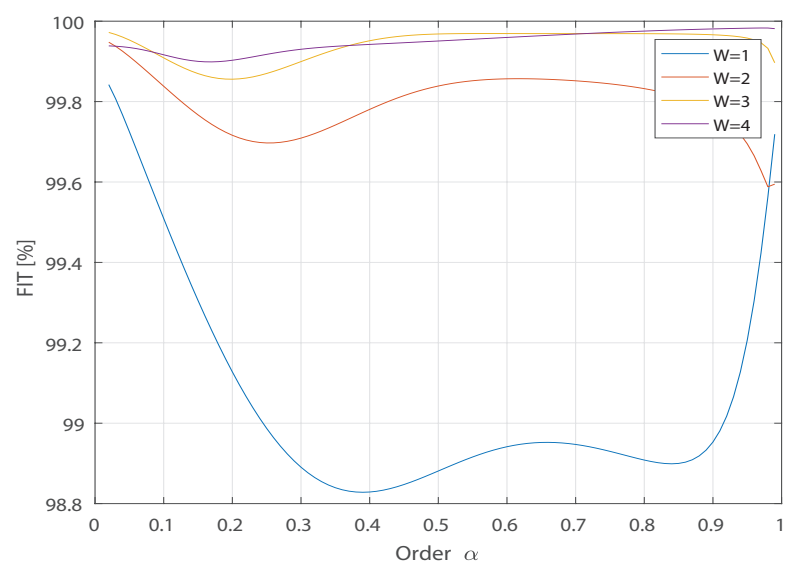

Fig. 5. The fitting of approximated module $M_{O R A}(j \omega)$ to analytical one (24) as a function of bandwidth $W$ for different orders $\alpha$

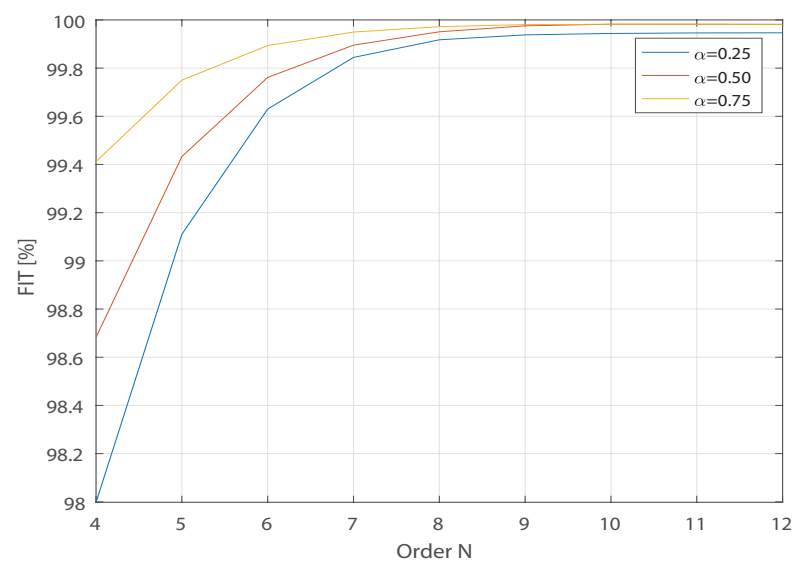

Fig. 6. The fitting of approximated module $M_{O R A}(j \omega)$ to analytical one (24) as a function of $N$ for different $\alpha, W=4$

\section{Conclusions}

The main conclusion from this paper is that the use of ORA allows us precisely describe the $\mathrm{AB}$ operator in the time and frequency domain.

However, the accuracy of the proposed, ORA based approximation strongly depends on non integer order $\alpha$. The recommended range to use it is for $0.5<\alpha<1.0$, because approximation in the range $0.0<\alpha<0.5$ is less accurate independently on order $N$. Increasing the bandwidth $W$ improves the accuracy for each value of order $\alpha$. This allows us conclude that the bandwidth $W$ should be always set as broad as possible to obtain the good accuracy independently on $\alpha$ and $N$. 
The further investigations will cover the extended theoretical analysis of the presented results, for instance calculation of analytical formula of the approximated step response (19), deeper accuracy and convergence analysis is also planned.

\section{Acknowledgment}

This paper was sponsored by AGH project no 16.16.120.773.

\section{References}

[1] Atangana, A., \& Baleanu, D. (2016). New fractional derivatives with nonlocal and non-singular kernel: theory and application to heat transfer model. Thermal Science, 20, 2, 763-769.

[2] Baleanu, D., \& Fernandez, A. (2017). On some new properties of fractional derivatives with Mittag-Leffler kernel. arXiv:1712.01762v1 [math.CA] 5 Dec 2017.

[3] Sene, N. (2019). Analytical solutions of Hristov diffusion equations with non-singular fractional derivatives. Chaos 29, 023112; https://doi.org/10.1063/1.5082645.

[4] Gomez, J.F., Torres, L., \& Escobar, R.F. (2019). Fractional Derivatives with Mittag-Leffler Kernel Trends and Applications in Science and Engineering. Studies in Systems, Decision and Control, vol. 194, Springer.

[5] Oprzedkiewicz, K. (2016). Accuracy estimation of digital fractional order PID controller. Theory and applications of non-integer order systems. 8th Conference on Non-integer order calculus and its applications: [20-21 September 2016], Zakopane, Poland. Eds. A. Babiarz et al. Springer International Publishing, cop. 2017 (Lecture Notes in Electrical Engineering; ISSN 1876-1100; vol. 407), ISBN: 978-3-319-45473-3; e-ISBN: 978-3-319-45474-0, pp. 265-275.

[6] Oprzedkiewicz, K., Mitkowski, W., \& Gawin, E. (2016). An estimation of accuracy of Oustaloup approximation. Challenges in automation, robotics and measurement techniques: proceedings of AUTOMATION-2016, March 2-4, 2016, Warsaw, Poland. Eds. R. Szewczyk, C. Zieliński, M. Kaliczyńska. Springer International Publishing, cop. 2016. (Advances in Intelligent Systems and Computing; ISSN 2194-5357; vol. 440), ISBN: 978-3-319-29356-1; e-ISBN: 978-3-31929357-8, pp. 299-307.

[7] Kaczorek, T. (2011). Selected Problems in Fractional Systems Theory. Springer Verlag.

[8] Kaczorek, T., \& Rogowski, K. (2014). Fractional Linear Systems and Electrical Circuits. Bialystok University of Technology.

[9] Oustaloup, A., Levron, F., Mathieu, B., \& Nanot, F.M. (2000). Frequency-band complex noninteger differentiator: characterization and synthesis. IEEE Transactions on Circuits and Systems I: Fundamental Theory Applications, 47, 1, 25-39.

[10] Caponetto, R., Dongola, G., Fortuna, 1., \& Petras, I. (2010), Fractional Order Systems. Modeling and Control Applications. World Scientific Series on Nonlinear Science, Series A, vol. 72, World Scientific Publishing. 